\title{
Técnicas de análisis estadístico multivariado: Una aplicación para el sector bancario hondureño
}

\author{
Omar Bonerge Pineda Lezama ${ }^{1}$ \\ Rafael Gómez Dorta \\ Universidad Tecnológica Centroamericana, San Pedro Sula, Honduras.
}

(Recibido: Abril, 2016. Aceptado para publicación: Diciembre, 2016)

\section{Resumen:}

Este trabajo presenta un procedimiento para la medición, el análisis y la identificación de oportunidades de mejora de la calidad de los servicios en el sector bancario hondureño, así como la determinación del Índice de Satisfacción del Cliente (ISC) a partir de la identificación de las variables críticas de calidad de dichos servicios. El procedimiento utilizado toma en cuenta el concepto de calidad del servicio y los modelos de medición más reconocidos e integra elementos de: investigación cualitativa, estadística multivariada y calidad. Se realiza el análisis de la calidad con una óptica integral, lo que permite conocer cuáles son las dimensiones de la calidad del servicio analizado y establecer un estado comparativo con las investigaciones realizadas. Se determina cuáles son los atributos que discriminan en el estado de satisfacción del cliente, lo que permite mantener un estado de control sobre dichas variables. Se utilizan como técnicas estadísticas fundamentales, el análisis descriptivo, el análisis factorial y el análisis discriminante, con el uso de los Software SPSS 17.0 y el STATGRAPHICS CENTURION. Como resultado final se concluye que los clientes evalúan a los servicios del banco en base a tres dimensiones proyectadas (conveniencia, capacidad de respuesta y visibilidad) y por tanto el banco debe sobresalir en ellas para proyectar una buena imagen. Además, existen cinco variables que discriminan en cuanto a la satisfacción del cliente a las cuales se debe prestar la debida atención para un índice de satisfacción elevado.

Palabras Claves: Calidad de servicio, Estadística multivariada, Análisis discriminante, Sector bancario.

\begin{abstract}
:
This paper presents a method for the measurement, analysis and improvement of quality services in the Honduran banking sector and determining the Customer Satisfaction Index (CSI) from the identification of critical variables of the quality of these services. The procedure considers the concept of quality of service and the most recognized measurement models and integrates the following elements: qualitative research, multivariate statistics and quality. Analysis with an integrated optical quality is done, allowing to know what the dimensions of service quality are and establishes a comparative analyzed with state investigations. It determines which attributes discriminate the state of customer satisfaction are, thus maintaining a state of control over these variables. They are used as basic statistical techniques, descriptive analysis, factor analysis and discriminant analysis, using the SPSS 17.0 software and STATGRAPHICS CENTURION. As a result, it is concluded that customers evaluate the services of the bank based on three-dimensional projected (convenience, responsiveness and visibility) and therefore the bank should stand on them to project a good image. In addition, there are five variables that discriminate in terms of customer satisfaction which should be given due attention for a high satisfaction rate.
\end{abstract}

${ }^{1}$ Autor para correspondencia. Email: omarpineda@unitec.edu 
Keywords: Quality of service, Multivariate statistics, Discriminant analysis, Banking sector

\section{Introducción}

Dado el aumento de los niveles de competitividad en el contexto mundial y hondureño, las instituciones financieras necesitan reconocer la importancia de atender correctamente las necesidades de sus clientes, con el propósito de alcanzar relaciones a largo plazo con él y lograr mantenerse vigentes en el mercado, es decir, se hace necesario identificar todas las variables que influyen y determinan el comportamiento de los clientes. Así, identificar los factores que afectan a la calidad percibida por los clientes, los elementos que producen su satisfacción y consecuente lealtad hacia una determinada empresa, se hace muy necesario.

La evaluación de la satisfacción de los clientes debe ser un objetivo esencial en cualquier organización de servicios. Hay varios cuestionarios que han sido utilizados con este propósito, sin embargo, hay estudios que han reportado problemas en su uso (Van Dyke,1999). Para evaluar la opinión del cliente de forma clara se requiere diseñar un cuestionario apropiado, aplicarlo eficientemente y analizar sus resultados con profundidad; sin embargo, en la mayoría de los casos el análisis se limita a calcular las frecuencias de las respuestas de las diferentes preguntas, lo cual no es desarrollar un enfoque limitado, pues los cuestionarios van a tener muchas preguntas correlacionadas. Esto justifica la necesidad de utilizar las Técnicas estadísticas apropiadas, que faciliten conocer mejor la información que nos brindan los cuestionarios.

Esta investigación pretende identificar las dimensiones que determinan la calidad percibida por los clientes de un banco, para comprender de qué manera estos producen satisfacción hacia una determinada institución bancaria. Aplicando técnicas estadísticas multivariadas se estimarán las dimensiones de la calidad percibida del servicio bancario, se identificarán las variables que discriminan en cuanto a el estado de satisfacción y sobre esta base se propone un método novedoso que permite estimar un índice de satisfacción del cliente, el cual será usado para monitorear el avance del proceso de mejoramiento de dicha satisfacción.

Para ello, la presente investigación plantea una estructura teórica que relaciona diferentes ítems y factores subyacentes, confirmando la misma a través de un Análisis Factorial, mediante la técnica de Análisis de Componentes Principales (ACP). Para poder determinar el índice de satisfacción, se hace necesario identificar aquellas variables que discriminan sobre el estado de dicha satisfacción para lo cual se utilizará la técnica de análisis Discriminante. Así, el trabajo pretende añadir valor tanto desde el punto de vista académico como para la gestión de entidades bancarias, lo anterior a través de la identificación de los factores determinantes de la calidad percibida del servicio prestado y la determinación del indicador de satisfacción que permitirá evaluar sistemáticamente el programa de mejora de la satisfacción del banco. 


\section{Planteamiento de la investigación}

Uno de los principales problemas que actualmente presentan las empresas hondureñas, principalmente las instituciones de carácter financiera-bancarias, es el manejo y manipulación efectiva de datos, que le proporcionen mayor claridad en todas sus operaciones y decisiones. A consideración de los autores aún no se ha desarrollado debidamente el potencial de resultados que estas nos podrían proporcionar, principalmente en el área financiera-bancaria.

Las actividades orientadas a la retención de los clientes generan una serie de beneficios a las empresas, por lo tanto, es necesario identificar los factores o dimensiones que afectan la satisfacción del cliente de una institución financiera, pues esto constituye un modo de consolidar una cartera de clientes y trazar planes de acción que conduzcan a mejoras significativas para la organización. Este trabajo tiene como base los trabajos desarrollados por diferentes autores referidos al marketing bancario (García de Madariaga y Pita, 2001; Malhotra, 2004) y otros referidos al mejoramiento de la calidad de los servicios bancarios (Pita Castelo, 2004; Salkey, 2007).

En la revisión de la literatura no se encontraron evidencias de la existencia de un procedimiento científicamente fundamentado que permita medir la satisfacción del cliente bancario. Por lo tanto, el problema de investigación queda formulado bajo la pregunta siguiente: ¿Cómo desarrollar un procedimiento que permita identificar las dimensiones fundamentales de la calidad de los servicios bancarios y definir las variables claves que determinan la satisfacción del cliente?, A partir de esto se definen los objetivos de la investigación como:

\subsection{Objetivo General}

Diseñar un procedimiento que permita la medición, el análisis y la identificación de oportunidades de mejora de los servicios bancarios.

\subsection{Objetivos Específicos}

- Identificar las dimensiones de la calidad de los servicios bancarios y efectuar comparación con los estudios identificados en la literatura.

- Determinar las variables que discriminan y permiten la medición de la satisfacción del cliente.

- Determinar el Índice de Satisfacción del Cliente de los servicios bancarios.

\section{El concepto de la calidad del servicio}

El concepto de calidad se extendió a todas las fases de la vida de un producto o servicio, desde su concepción o diseño hasta su fabricación y posterior uso por parte del cliente, siendo el lema "Cero Defectos". En la actualidad los productos y servicios no sólo tienen que ser aptos para el uso que se les ha asignado, sino que además tienen que igualar e incluso superar 
las expectativas que los clientes han depositado en ellos. El objetivo consiste en satisfacer a los clientes desde el principio hasta el fin. Esta nueva concepción de la calidad es lo que se conoce como "Calidad del Servicio".

Inicialmente se centra sobre la calidad de la realización del servicio y sobre su adaptación a las especificaciones fijadas por los prestatarios, abordándose el tema de la calidad en el servicio como si se tratara de calidad de un producto (Grönroos, 1994). Después el concepto se desplaza hacia el consumidor, vinculándose de alguna forma a la noción del nivel de satisfacción que es objeto de la realización del servicio, produciéndose de esta forma una mutación, pasando de una calidad objetiva a una calidad subjetiva. El concepto se entiende de esta última forma en base a la calidad percibida o humanística frente a la calidad técnica o mecánica inicial. Introduciendo la percepción, la definición es desde la óptica del cliente.

Esta forma de entender el concepto es soportada ampliamente y es la base del desarrollo teórico y metodológico que permite modelar el proceso de calidad de servicio propuesto por Parasuraman, Zeithaml y Berry (1993). Los clientes valoran la calidad del servicio al comparar lo que desean y esperan, con lo que actualmente perciben. Ellos determinaron que la percepción de la calidad de un servicio posee diez componentes: cortesía, comunicación, comprensión, credibilidad, veracidad, capacidad de respuesta, competencia, accesibilidad, seguridad y tangibilidad.

\subsection{Los modelos conceptuales para medir la calidad de servicio}

Un modelo de calidad del servicio no es más que una representación simplificada de la realidad, que toma en consideración aquellos elementos básicos capaces por sí solos de explicar convenientemente el nivel de calidad alcanzado por una organización desde el punto de vista de sus clientes. Numerosos modelos (Grönroos, 1994; Parasuraman, Zeithaml y Berry, 1993; Cronin \& Taylor, 1992) se han desarrollado para comprender la definición y formación de las percepciones sobre calidad de servicios.

El modelo más comprehensivo de todos los revisados es el SERVQUAL, fue desarrollado como consecuencia de la ausencia de literatura que tratase específicamente la problemática relacionada con la medida de la calidad del servicio, es un instrumento de medición del constructo calidad del servicio, el mismo está basado en el modelo teórico de los GAPS. Sin embargo, y aunque esta escala de medición ha tenido un impacto muy importante, tampoco ha estado exenta de críticas, que cubren tanto aspectos conceptuales como operativos. Dentro de este contexto, diversos trabajos han aparecido en la literatura defendiendo y replicando al modelo SERVQUAL (Carman, 1990; Cronin y Taylor, 1992), a partir de los cuales se propuso un instrumento de medición alternativo, la escala SERVPERF (Cronin y Taylor, 1992), la cual se compone de los 22 ítems de la escala SERVQUAL, pero utilizados solo para medir las percepciones del servicio.

La revisión literaria realizada permitió entender los conceptos de servicio y calidad del servicio, lo cual es fundamental para comprender el objeto y las características de los modelos 
de medición. En las revisiones realizadas se pudo comprobar que el concepto calidad, está íntimamente asociado a la satisfacción del cliente. Para Larrea (1991) existen cinco niveles en la satisfacción: excitación, satisfacción, irritación, insatisfacción y enfado.

Consecuentes con lo anterior, evaluar la calidad de los servicios bancarios, puede interpretarse como evaluar el grado en el que se satisfacen las necesidades de los clientes, pero para esto se requiere identificar en ellos, cuáles son las necesidades importantes, también conocidas como dimensiones de calidad. La mayoría de los clientes utilizan según él, cinco dimensiones para llevar a cabo dicha evaluación, que son: fiabilidad, seguridad, capacidad de respuesta, empatía e intangibilidad. Malhotra (2004), establece concretamente cuatro dimensiones para los servicios bancarios, las cuales son: capacidad de respuesta, conveniencia, visibilidad y competencia. Estas dimensiones, van a servir de punto de referencia a la hora de realizar la valoración objeto del presente trabajo.

\section{Hipótesis}

$H_{1}$ : Las variables que definen la satisfacción del cliente de los servicios bancarios, están relacionadas básicamente con la capacidad de respuesta de la organización.

\section{Descripción del instrumento}

Para la elaboración del cuestionario para la medición de la calidad del servicio se empleó el método de los incidentes críticos, entendiéndose como incidente crítico, un ejemplo específico del desempeño organizacional desde la perspectiva del usuario. En este método, luego de colectar los incidentes críticos, se forman grupos y se redactan frases que reflejen el contenido de los mismos, las cuales se denominan elementos de satisfacción, los cuales serán los puntos considerados en el cuestionario. El estudio de los incidentes críticos para los servicios bancarios ha sido desarrollado en investigaciones precedentes (Salkey, 2007).

Teniendo en cuenta estos estudios se diseñó el cuestionario para evaluar la calidad del servicio, el cual fue sometido a revisión de expertos externos para verificar el cumplimiento de requisitos de redacción, entendimiento y propósito de la medición. El mencionado instrumento consta de 13 ítems del servicio medidos en un escalamiento tipo Likert, que va desde totalmente satisfecho, hasta totalmente insatisfecho. Los primeros doce ítems corresponden a los elementos de satisfacción definidos en el análisis de incidentes críticos y, se agregó un último ítem, que hace referencia a la satisfacción general percibida por cada cliente ("está usted totalmente satisfecho o insatisfecho, después de haber recibido el servicio“), el cual se empleará posteriormente para formar los grupos y analizar variables críticas, así como sus relaciones con las dimensiones de calidad.

\section{Procedimiento para el análisis de calidad de los servicios bancarios}

El procedimiento elaborado se basa en los criterios definidos por Pérez (2007), y se presenta en la figura No. 1, como se puede apreciar el mismo toma en cuenta uno de los aspectos fundamentales de la investigación cuantitativa, que es el desarrollo de instrumentos de 
medición completamente confiables, válidos y objetivos. El procedimiento organiza metodológicamente las actividades a realizar.

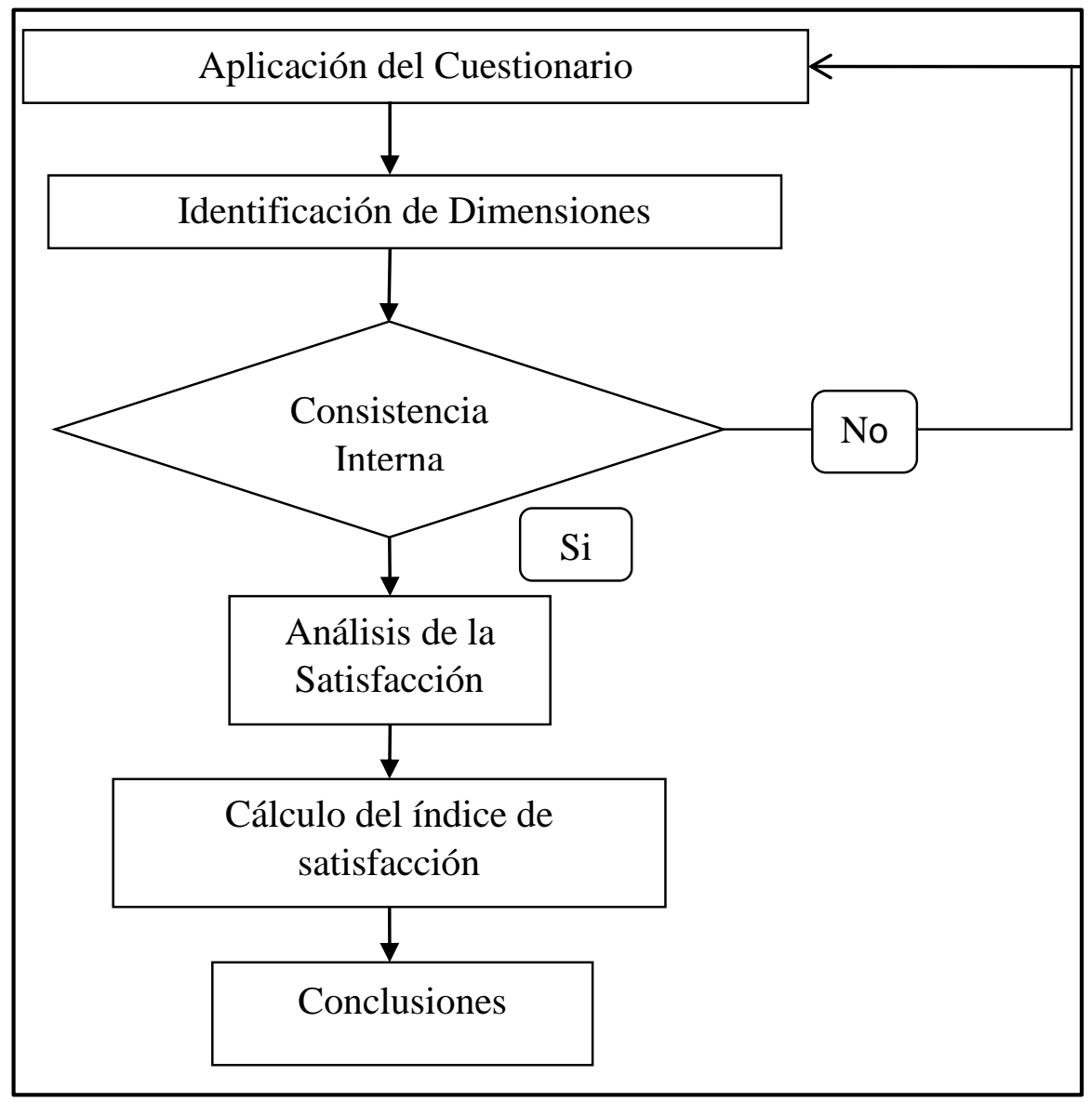

Figura No. 1 Procedimiento para el análisis y medición de calidad de los servicios bancarios

\section{Resultados y hallazgos de la investigación}

En este capítulo se detalla la aplicación etapa por etapa del procedimiento propuesto, destacando la aplicación de cada una de las herramientas de análisis y las conclusiones a que se arriban en cada uno de los análisis.

\subsection{Aplicación del cuestionario}

Para obtener datos sobre la percepción de los clientes frente a la satisfacción de sus necesidades, que permitan identificar con claridad, la estructura de las dimensiones de calidad, y al mismo tiempo, estimar el nivel de satisfacción del servicio, el cuestionario elaborado se aplicó a un grupo de clientes que, durante el mes de agosto de 2015, recibieron el servicio en uno de los bancos de la ciudad de San Pedro Sula. En vistas de que en tal período un mismo usuario podría recibir varios servicios, se realizó una sola encuesta por 
usuario, a fin de garantizar que las percepciones capturadas sobre la calidad durante los servicios prestados fuesen independientes entre sí. Otra razón fue la de evitar generar malestar al cliente realizándole más de una encuesta, lo que, de hacerse, aumentaría la posibilidad de que este responda en las siguientes encuestas de una manera no razonada.

Se aplicó la encuesta a un total de 382 clientes, se anularon por diferentes problemas un total de 47 , por lo que se procesaron 335 , para un $88 \%$, por lo que el error fue de $5.3 \%$, lo que es considerado adecuado. La encuesta se realizó en los días de lunes a sábado, en el horario entre 2:00 p.m. a 5:00 p.m. Al banco llegaban por lo menos 40 clientes diarios. A cada cliente se le asignó un número del 1 al 40. Se generó una lista de 15 números aleatorios diferentes entre 1 y 40, cada día. Lo cual comprueba que la elección se realizó a través de un procedimiento de muestreo aleatorio simple. A partir de aquí se creó la matriz de datos correspondientes para la tabulación de los mismos.

\subsection{Identificación de las dimensiones de calidad}

Para determinar la cantidad de dimensiones de calidad que subyacen en los elementos sometidos a valoración, se debe emplear inicialmente la técnica estadística de Análisis de Componentes Principales (Dallas, 1998). Para seleccionar el método de extracción de factores se tendrán en cuenta los criterios establecidos por Malhotra (2004). Con el fin de facilitar la interpretación de las dimensiones y determinar la estructura de cada una de ellas se debe proceder a aplicar el método de rotación Varimax, considerándose como cargas importantes, aquellas con valor absoluto superior a 0,4 (Dallas, 1998). Con esto se busca que las cargas de cada elemento original estén cercanas a 1 en una sola dimensión y próximas a cero en las demás.

Tabla 1. Cálculo del KMO y Resultados de la Prueba de Bartlett

\begin{tabular}{|cl|r|}
\hline \multicolumn{2}{|l|}{$\begin{array}{l}\text { Medidas de adecuación muestral de Kaiser- } \\
\text { Meyer-Olkin }\end{array}$} & .895 \\
\hline & Chi-Cuadrado & 1093.1 \\
Prueba de & Aproximado & 94 \\
esfericidad & Grados de Libertad & 66 \\
Bartlett's & Nivel de Significancia & .000 \\
& & \\
\hline
\end{tabular}

En la tabla 1 se presentan los resultados alcanzados al aplicar las pruebas fundamentales para valorar la pertinencia del Análisis Factorial. Se aprecia que el índice KMO es igual a 0.895, el valor chi-cuadrado es 1093 con un valor de significación de 0.000 , además se obtuvo un valor bajo del determinante de la matriz de correlaciones (0.036), todos constituyen 
resultados favorables según diferentes autortes, Malhotra (2004); Hair (1999), lo que permite concluir que el análisis factorial resulta pertinente y puede proporcionar conclusiones satisfactorias.

\subsection{Extracción de factores}

En la tabla 2 se muestran todos los factores que se obtienen con el método de componentes principales. Solo hay dos factores con valor propio inicial superior a uno, aunque hay un tercer factor relativamente cerca. El primer factor explica el $43 \%$ de la varianza de la muestra y entre los tres explican el $68 \%$, lo que puede interpretarse como un porcentaje aceptable según Malhotra (2004). Entonces podemos expresar cada una de nuestras variables como una combinación lineal de estos tres factores o variables latentes.

Tabla 2. Varianza Total Explicada

\begin{tabular}{|c|c|c|c|c|c|c|}
\hline \multirow{2}{*}{ Componente } & \multicolumn{3}{|c|}{ Valores Propios Iniciales } & \multicolumn{3}{|c|}{$\begin{array}{c}\text { Sumas de las saturaciones al } \\
\text { cuadrado } \\
\text { de la extracción }\end{array}$} \\
\cline { 2 - 7 } & Total & $\begin{array}{c}\% \text { de } \\
\text { Varianza }\end{array}$ & acumulativo \% & Total & $\begin{array}{c}\% \text { de } \\
\text { varianza }\end{array}$ & acumulativo \% \\
\hline 1.000 & 4.541 & 43.124 & 43.124 & 4.541 & 43.124 & 43.124 \\
\hline 2.000 & 1.158 & 15.135 & 58.259 & 1.158 & 15.135 & 58.259 \\
\hline 3.000 & 0.915 & 10.022 & 68.281 & 0.915 & 10.022 & 68.281 \\
\hline 4.000 & 0.870 & 6.251 & 74.273 & & & \\
\hline 5.000 & 0.757 & 5.011 & 79.284 & & & \\
\hline 6.000 & 0.695 & 4.500 & 83.784 & & & \\
\hline 7.000 & 0.629 & 4.111 & 87.895 & & & \\
\hline 8.000 & 0.585 & 3.700 & 91.595 & & & \\
\hline 9.000 & 0.534 & 3.440 & 95.035 & & & \\
\hline 10.000 & 0.480 & 2.007 & 97.042 & & & \\
\hline 11.000 & 0.449 & 1.600 & 98.642 & & & \\
\hline 12.000 & 0.387 & 1.358 & 100.000 & & & \\
\hline
\end{tabular}

\subsection{Rotación de factores}

La finalidad de la rotación es ayudarnos a interpretar el sentido y significado de los factores extraídos. En la tabla 3 se presentan las dimensiones de la calidad del servicio, la carga de los elementos luego de efectuar la rotación Varimax y el porcentaje de varianza explicado por cada dimensión. Se consideraron como cargas significativas, aquellas con valor absoluto superior a 0.4 (Dallas, 1998). En este caso se determinó que la calidad de servicio del banco, está en función de tres dimensiones de calidad, con una explicación del $68 \%$ de la varianza total. Este porcentaje se considera satisfactorio para los fines del estudio, teniendo presente 
la subjetividad que caracteriza las percepciones de los usuarios y los factores incontrolables que intervienen en ellas. Además, esta cantidad de dimensiones es coherente con lo planteado por la literatura para los servicios bancarios, lo que evidencia la validez del constructo estudiado.

\subsection{Consistencia interna}

Con el propósito de analizar el carácter confiable de la estructura de las dimensiones de calidad y del cuestionario completo, se recurrió al coeficiente de consistencia interna Alpha de Cronbach $(\alpha)$, Sánchez (1995). Obteniéndose un valor $\alpha=0.846$, ver tabla 4. Este resultado es satisfactorio de acuerdo a lo que plantea la literatura antes citada. El valor del coeficiente alpha si el ítem es eliminado se mantiene en valores alrededor de 0.83 lo que indica alta confiablidad y correlación de todos los ítems incluidos.

Tabla 3. Estructura de las Dimensiones de Calidad

\begin{tabular}{|c|c|c|c|c|}
\hline No. & Dimensión & Definición & Elementos & Carga \\
\hline \multirow{4}{*}{1} & \multirow{4}{*}{ Visibilidad } & \multirow{4}{*}{$\begin{array}{ll}\text { Grado hasta el cual } & \text { se } \\
\text { demuestra transparencia } & y \\
\text { presentación para el cliente. } & \end{array}$} & Transacciones legibles & 0.38 \\
\hline & & & Atractivo de las instalaciones & 0.609 \\
\hline & & & Capacidad de los empleados & 0.66 \\
\hline & & & Apariencia y porte & 0.768 \\
\hline \multicolumn{5}{|c|}{ 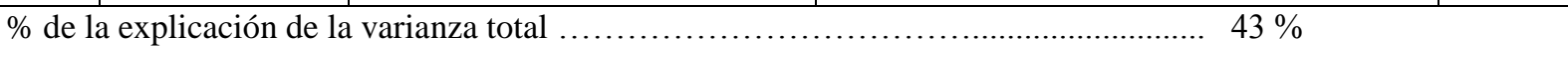 } \\
\hline \multirow{5}{*}{2} & \multirow{5}{*}{$\begin{array}{l}\text { Capacidad de } \\
\text { respuesta }\end{array}$} & \multirow{5}{*}{$\begin{array}{l}\text { El empleado del banco reacciona } \\
\text { a tiempo, se comporta de manera } \\
\text { afable, dedicada y demuestra } \\
\text { capacidad mientras trabaja con el } \\
\text { cliente. }\end{array}$} & Servicio cordial y personalizado & 0.632 \\
\hline & & & Facilidad de conseguir préstamo & 0.821 \\
\hline & & & Rapidez de servicio & 0.491 \\
\hline & & & Habilidad de cumplir servicio & 0.612 \\
\hline & & & Cooperación con los clientes & 0.634 \\
\hline \multicolumn{5}{|c|}{ 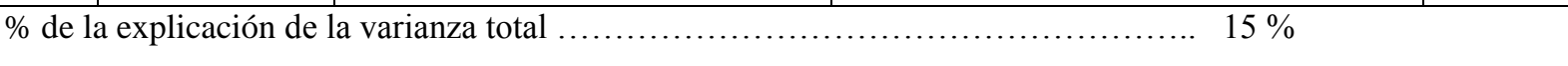 } \\
\hline \multirow{3}{*}{3} & \multirow{3}{*}{ Conveniencia } & \multirow{3}{*}{$\begin{array}{l}\text { El banco se presta en una hora y } \\
\text { lugar conveniente para el cliente }\end{array}$} & Ubicación adec. del banco & 0.768 \\
\hline & & & Ubicación adecuada del cajero & 0.675 \\
\hline & & & Conveniencia de Horario & 0.592 \\
\hline \multicolumn{5}{|c|}{ 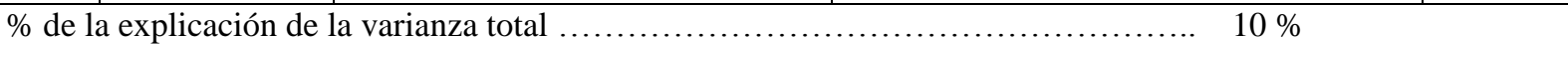 } \\
\hline & & $\overrightarrow{\mathrm{at}}$ & $68 \%$ & \\
\hline
\end{tabular}




\subsection{Análisis de la satisfacción del cliente}

Es importante entonces seleccionar las variables independientes que permiten diferenciar los dos grupos creados (satisfechos y no satisfechos), para evitar esfuerzos de mejora que no conduzcan a resultados favorables, para esto se va a utilizar la técnica de Análisis Discriminante. Al aplicar la misma se podrá seleccionar aquellas variables claves que discriminan significativamente para la predicción de la satisfacción del cliente (su estado de pertenencia a uno de los grupos).

Tabla 4. Determinación del Coeficiente Alpha de Cronbach

\begin{tabular}{|l|c|c|c|c|}
\hline & $\begin{array}{c}\text { Escala Media } \\
\text { de Ítems } \\
\text { Eliminados }\end{array}$ & $\begin{array}{c}\text { Escala de } \\
\text { Varianza de } \\
\text { Ítem } \\
\text { Eliminados }\end{array}$ & $\begin{array}{c}\text { Correlación } \\
\text { Total de Ítem } \\
\text { Corregidos }\end{array}$ & $\begin{array}{c}\text { Alpha de } \\
\text { Cronbach's } \\
\text { de Ítems } \\
\text { Eliminados }\end{array}$ \\
\hline Servicio cordial y personalizado & 40.093 & 67.695 & 0.581 & 0.829 \\
\hline Transacciones legibles & 39.863 & 69.813 & 0.471 & 0.837 \\
\hline Facilidad de conseguir prestamos & 40.707 & 70.866 & 0.379 & 0.844 \\
\hline Ubicación adecuada del banco & 39.612 & 71.909 & 0.372 & 0.843 \\
\hline Ubicación adecuada de los cajeros & 39.976 & 68.622 & 0.483 & 0.836 \\
\hline Rapidez del servicio & 40.684 & 65.409 & 0.586 & 0.828 \\
\hline Conveniencia de horario del banco & 40.188 & 68.932 & 0.432 & 0.840 \\
\hline Atractivo de las instalaciones físicas & 40.093 & 69.755 & 0.469 & 0.837 \\
\hline Capacidad de los empleados & 40.179 & 67.632 & 0.610 & 0.827 \\
\hline Apariencia y porte de los empleados & 39.872 & 69.711 & 0.508 & 0.834 \\
\hline $\begin{array}{l}\text { Habilidad de cumplir el servicio } \\
\text { prometido }\end{array}$ & 40.209 & 66.256 & 0.627 & 0.825 \\
\hline Cooperación con los clientes & 40.260 & 65.331 & 0.638 & 0.824 \\
\hline
\end{tabular}

Alfa de Cronbach General

0.846

Se realizó la prueba M. de Box, los resultados se presentan en la figura 2, estos confirman que las matrices de varianzas-covarianzas son diferentes, lo que evidencia el cumplimiento de los supuestos para aplicar la técnica propuesta. En la tabla 5 se presentan los valores de los estadísticos más importantes en el análisis. El auto valor de 1,0822 y la correlación canónica de 0,7209 permiten extraer una primera conclusión: existe una única función discriminante que permite de forma significativa (sig. 0.000) clasificar a los sujetos en los dos grupos: satisfechos y no satisfechos. El valor de lambda de Wilks de 0.4802 permite extraer una segunda conclusión: aunque la función discriminante servirá para pronosticar la pertenencia a los grupos, seguramente no todas las variables serán discriminantes, su valor 
denota ciertas similitudes entre los grupos. por lo que se debe estudiar la influencia de cada una de las variables en la función discriminante.

\begin{tabular}{|c|c|c|}
\hline \multicolumn{2}{|c|}{ M de Box } & 78.316 \\
\hline $\mathrm{F}$ & Aprox. & 7.638 \\
\hline & gl1 & 10 \\
\hline & $\mathrm{gl} 2$ & 45869.024 \\
\hline & Sig. & .000 \\
\hline
\end{tabular}

Figura 2. Test M. de Box

Tabla 5. Estadísticos Fundamentales del Análisis Discriminante Realizado

\begin{tabular}{|l|l|l|l|l|}
\hline $\begin{array}{l}\text { Función } \\
\text { Discriminante }\end{array}$ & Auto valor & Porcentaje relativo & \multicolumn{2}{|l|}{ Correlación canónica } \\
\hline 1 & 1.08227 & 100.00 & 0.72094 & \\
\hline $\begin{array}{l}\text { Funciones } \\
\text { derivadas }\end{array}$ & Lambda de Wilks & Chi-cuadrada & Grados de libertad & Valor-P \\
\hline 1 & 0.480246 & 239.8407 & 12 & 0.0000 \\
\hline
\end{tabular}

El proceso a seguir es realizar la prueba con todas las variables mediante la opción "usar método de inclusión por pasos", para, posteriormente eliminar las no significativas en la función. La tabla 6 indica los pasos seguidos para la construcción de la función discriminante y consecuentemente que variables independientes son significativas para el modelo. En este caso las variables significativas son: 1) Servicio cordial y personalizado $\left(\mathrm{S}_{1}\right)$; 2$)$ Habilidad de cumplir el servicio prometido $\left.\left(\mathrm{S}_{11}\right) ; 3\right)$ Rapidez del servicio $\left(\mathrm{S}_{6}\right)$; 4) Atractivo de las instalaciones $\left(\mathrm{S}_{8}\right)$ y Capacidad de los empleados $\left(\mathrm{S}_{9}\right)$.

Tabla 6. Análisis Discriminante: Regresión Por Pasos, Método Hacia Adelante

\begin{tabular}{|c|c|c|c|c|c|c|}
\hline Paso & $\begin{array}{c}\text { Agregando } \\
\text { variables }\end{array}$ & $\begin{array}{c}\text { F para } \\
\text { introducir }\end{array}$ & $\begin{array}{c}\text { Lambda } \\
\text { de Wilk }\end{array}$ & F aproximada & Valor-P & Comentario \\
\hline 1 & S1 & 211.943 & 0.611073 & 211.943 & 0.0000 & 1 variable en el modelo \\
\hline 2 & S6 & 39.7791 & 0.54569 & 138.202 & 0.0000 & 2 variable en el modelo \\
\hline 3 & S11 & 16.8786 & 0.519214 & 102.167 & 0.0000 & 3 variable en el modelo \\
\hline 4 & S 8 & 8.68008 & 0.505907 & 80.5734 & 0.0000 & 4 variable en el modelo \\
\hline 5 & S9 & 5.9008 & 0.496993 & 66.5962 & 0.0000 & $\begin{array}{c}5 \text { variable en el modelo ( } \\
\text { Modelo final seleccionado) }\end{array}$ \\
\hline
\end{tabular}


La función discriminante queda definida como:

Satisfacción $=0.6786 * S_{1}+0.3262 * S_{11}+0.406 * S_{6}-0.2874 * S_{8}+0.2084 * S_{9}$

Como se puede apreciar de las cinco variables seleccionadas, tres de ellas pertenecen a la dimensión de capacidad de respuesta, lo que evidencia el nivel de atención que hay que prestarle a la misma, si se quiere elevar el índice de satisfacción. En la tabla 7 se presenta el cuadro de la clasificación de los sujetos a partir de la aplicación de la función discriminante obtenida. En este caso se han clasificado correctamente el $87 \%$ de los casos, por lo que resulta confiable utilizar esta función para predecir el grupo en que clasifican los clientes y sobre esta base estimar la proporción de clientes satisfechos, la cual constituiría una buena estimación de la satisfacción del cliente.

Tabla 7. Clasificación De Los Sujetos A Partir De La Función Discriminante

\begin{tabular}{|c|c|c|c|}
\hline Actual satisfacción & Tamaño de grupo & $\begin{array}{c}\text { Predicho } \\
\mathbf{1}\end{array}$ & $\begin{array}{c}\text { Satisfacción } \\
\mathbf{2}\end{array}$ \\
\hline 1 & 226 & 198 & 28 \\
\hline & & $(87.61 \%)$ & $(12.39 \%)$ \\
\hline 2 & 109 & 14 & 95 \\
\hline & & $(12.84 \%)$ & $(87.16 \%)$ \\
\hline
\end{tabular}

\subsection{Determinación del Índice de Satisfacción del Cliente.}

Esta proporción se estimaría a partir de la expresión:

$I S C_{i}=\frac{N C S_{i}}{T C_{i}} * 100 \%$

donde:

$I S C_{i}$ : índice de satisfacción del cliente en el período i

$N C S_{i}$ : número de clientes clasificados como satisfechos por la función discriminante en el período i

$T C_{i}$ : Total de clientes encuestados en el período i. 
Tabla 8. Corte De Los Resultados De Evaluación De La Satisfacción

\begin{tabular}{|c|c|c|c|c|}
\hline Fila & Cliente & Grupo actual & Grupo Predicho & Probabilidad \\
\hline 336 & 1 & & 1 & 0.9891 \\
\hline 337 & 2 & & 1 & 0.7991 \\
\hline 338 & 3 & & 1 & 0.5951 \\
\hline 339 & 4 & & 2 & 0.644 \\
\hline 340 & 5 & & 2 & 0.7833 \\
\hline 341 & 6 & & 2 & 0.9028 \\
\hline 342 & 7 & & 1 & 0.8493 \\
\hline 343 & 8 & & 2 & 0.9951 \\
\hline 344 & 9 & & 2 & 0.9951 \\
\hline 345 & 10 & & 1 & 0.9902 \\
\hline . & . & & . & . \\
\hline 434 & 97 & & 1 & 0.9419 \\
\hline 435 & 98 & & 2 & 0.551 \\
\hline 436 & 99 & & 1 & 0.9256 \\
\hline 437 & 100 & & 1 & 0.8886 \\
\hline 438 & 101 & & 1 & 0.6306 \\
\hline 439 & 102 & & 1 & 0.9383 \\
\hline 440 & 103 & & 2 & 0.6034 \\
\hline 441 & 104 & & 1 & 0.9383 \\
\hline 442 & 105 & & 1 & 0.9383 \\
\hline 443 & 106 & & 2 & 0.9856 \\
\hline
\end{tabular}

Para validar el procedimiento se tomó una muestra de 106 clientes nuevos y se colocaron a partir de la fila 336 del fichero de datos, se dejó la celda de satisfacción en blanco para poder predecir el grupo de pertenencia del sujeto a partir de la función discriminante definida. Un corte del cuadro de los resultados se presenta en la tabla 8 . A partir de aquí se tiene que:

$\mathrm{NCS}_{1}=66 ; \mathrm{TC}_{1}=106$, luego sustituyendo en la ecuación dos se obtiene un índice de satisfacción de clientes de $62 \%$. Una estimación del intervalo de confianza al $95 \%$ para este indicador estaría dado por: $\mathrm{ISC}_{1}=[0.5232 ; 0.715]$, lo que significa que se tiene un nivel de confianza del $95 \%$ de que entre el 52 y el $71.5 \%$ de los clientes que reciben los servicios del banco en este período, están satisfechos. Este resultado indica un potencial de mejora elevado. 


\section{Conclusiones}

1. Los clientes evalúan los servicios de la institución bancaria estudiada de acuerdo con tres dimensiones básicas: conveniencia, capacidad de respuesta y visibilidad, resultado que es coherente con lo planteado por la literatura para los servicios bancarios.

2. Se determinaron las variables que más discriminan en cuanto al estado de satisfacción del cliente, estas son: servicio cordial y personalizado, rapidez del servicio, atractivo de las instalaciones, capacidad de los empleados y habilidad de cumplir el servicio prometido.

3. Se definió un método sencillo y no utilizado anteriormente para calcular el índice de satisfacción del cliente, al aplicarlo se determinó con un $95 \%$ de confianza que entre el 52\% y el $71.5 \%$ de los clientes se encuentran satisfechos, lo que indica que el banco objeto de estudio tiene un potencial de mejora elevado.

4. La evidencia empírica obtenida en este estudio muestra que tres de las cinco variables que discriminan en cuanto a satisfacción, tributan a la dimensión de capacidad de respuesta, lo que valida la hipótesis de investigación.

\section{Bibliografía}

Carman, J. M. (1990). Consumer Perceptions of Service Quality: An Assessment of the SERVQUAL Dimensions. Journal, of Retailing, 69 (Spring), 127-139.

Cronin, J., \& Taylor, S. (1992). Measuring Service Quality: A Reexamination and Extension. The Journal of Markting, 56 (3), 55-68.

Dallas, E. (1998). Métodos multivariados aplicados al análisis de datos. México: Thomson.

García de Madariaga, J. y Pita, J. (2001). Las claves de la satisfacción del cliente bancario, Cuadernos de información económica, 185, 115-125.

Grönroos, C. (1994). Marketing y gestión de servicios: la gestión de los momentos de la verdad y la competencia en los servicios. Madrid: Díaz de Santos.

Hair, Jr., J.F.; R.E. Anderson; R.L. Tatham y W.C. Black (1999): Análisis Multivariante. $5^{\text {a }}$ Ed. Madrid: Prentice Hall Iberia.

Larrea, P. (1991). Calidad de servicio del marketing a la estrategia. Madrid: Díaz De Santos.

Malhotra K., Naresh (2004). Investigación de Mercados. México D.F.: Pearson Prentice Hall.

Pérez, Jorge (2007). Evaluación y análisis de la calidad de un servicio de apoyo desde la perspectiva del usuario. Primer paso hacia la confiabilidad, VII, 40-55. 
Pita, J. (2004). La importancia y el comportamiento de cambio de los clientes bancarios: Un análisis empírico. Tribuna de economía, octubre-noviembre (818).

Salkey, M. (2007). Mejoramiento de la calidad de los servicios bancarios, aplicando filosofía lean-sixsigma. Tesis para optar por el título de Master en Ingeniería Industrial con mención en calidad. Cienfuegos: Universidad de Cienfuegos.

Sánchez, J.J.(1995). Manual de análisis de datos. Madrid: Alianza Textos.

Van Dyke, V. R. Prybutok and L.A. Kappelman (1999). Cautions on the use of the Servqual measure to assess the quality of information systems services. Decision Sciences. 30(3), 877-891.

Zeithaml Valarie A, Parasuraman A., Berry Leonard L. (1993). Calidad Total En La Gestión De Servicios. Bogota: Díaz de Santos.

"LA REVISTA INNOVARE NO SE HACE RESPONSABLE EN NINGÚN CASO DE LOS CONTENIDOS, DATOS, CONCLUSIONES U OPINIONES VERTIDAS EN LOS ARTÍCULOS PUBLICADOS, SIENDO ESTA RESPOSABILIDAD EXCLUSIVA DEL (DE LOS) AUTOR (AUTORES)" 